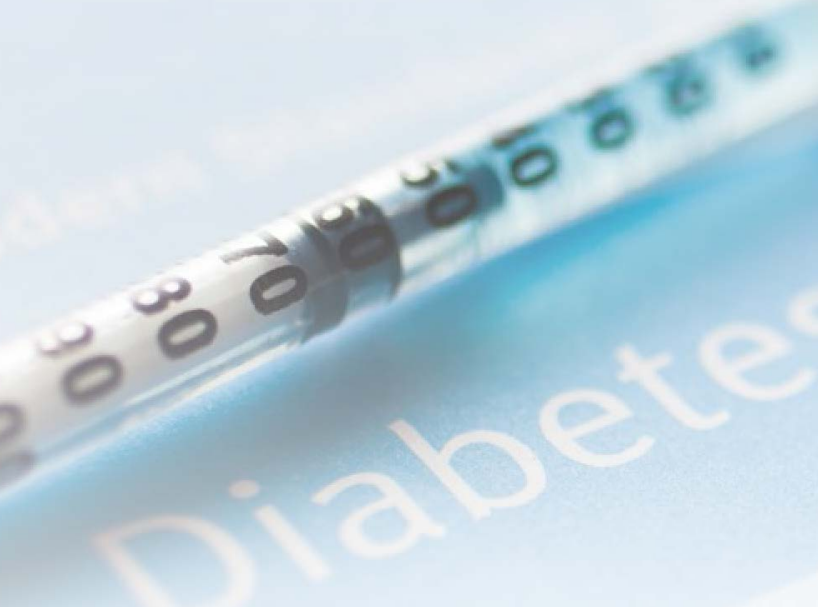

Diabetiker-Versorgung

\title{
Die Crux mit der Pauschale
}

\begin{abstract}
Offiziell können diabetologische Schwerpunktpraxen für die Mitbehandlung von Patienten zwar die Chronikerpauschale ansetzen. Tatsächlich müssen sie dafür aber in der Versorgung ein gutes Recall-System vorhalten.
\end{abstract}

Mit der Reform des hausärztlichen Kapitels im EBM hätte alles einfach werden sollen: Seit die Vertreterpauschale gestrichen wurde, wird für den ersten persönlichen Arzt-Patienten-Kontakt im hausärztlichen Versorgungsbereich die Versichertenpauschale abgerechnet. Dies unabhängig davon, ob es ein eigener Patient ist, ein Vertretungsfall oder aber auch ein Patient, der zur diabetologischen Mitbehandlung überwiesen wurde. Damit schien das Problem der diabetologischen Schwerpunktpraxen, die Chronikerpauschale nicht abrechnen zu können, gelöst zu sein. Denn auch im alten Hausarzt-EBM galt: Eine Chroniker-Pauschale darf nicht neben einer Vertreterpauschale berechnet werden. Doch der Schein trügt!

\section{Zwei mögliche Ziffern}

Die beiden neuen Chronikerpauschalen (EBM-Nrn. 03220 und 03221), die seit Oktober 2013 gelten, wurden für die aufwändige und umfangreiche Versorgung und Betreuung von Versicherten mit einer chronischen Erkrankung in den EBM eingeführt. Die Berechnung ist dabei natürlich weiterhin an bestimmte Voraussetzungen gebunden. Die erste und wichtigste Voraussetzung ergibt sich aus der Leistungslegende: „Zuschlag zu der Versichertenpauschale nach der Gebührenordnungsposition (GOP) 03000 für die Behandlung und Betreuung eines Patienten mit mindestens einer lebensverändernden chronischen Erkrankung“. Das trifft für Diabetes-Patienten in der Regel zu. Kritisch wird es jedoch bei der zweiten Abrechnungsvoraussetzung, der sogenannten 4-3-2-Regel: Denn innerhalb der letzten vier Quartale muss jeweils mindestens ein Arzt-Patienten-Kontakt pro Quartal in mindestens drei Quartalen wegen derselben gesicherten chronischen Erkrankung(en) und in derselben Praxis stattgefunden haben. Hierbei müssen in mindestens zwei Quartalen persönliche Arzt-Patienten-Kontakte stattgefunden haben - der Patient muss also tatsächlich vor Ort vom Arzt gesehen worden sein.

\section{Vorsicht mit 6-Monats-Rhythmus}

Diese Forderung wird aus der Praxiserfahrung heraus aber in den wenigsten Fällen erfüllt. Weil Patienten oft nur alle sechs Monate in der diabetologischen Praxis vorstellig werden. Konkret bedeutet dies: Der entsprechende Patient hat dann nur zwei Kontakte mit der Schwerpunktpraxis gehabt - damit kann die Chronikerpauschale nicht abgerechnet werden. Das ist insofern ärgerlich, weil diese Patienten entsprechend aufwändig sind. Hier hilft also nur ein gutes Recall-System und organisatorische Kompetenz. Werden solche Überweisungspatienten grundsätzlich zum Quartalsende einbestellt, wäre der notwendige 2. Kontakt im Folgequartal festzulegen. Damit könnten sich die diabetologischen Schwerpunktpraxen zumindest die Chronikerpauschale I (GOP 03220, 130 Punkte) sichern. Auch die Festlegung eines einfachen Kontrolltermins in einem weiteren (dritten) Quartal im Verlauf eines Jahres, würde in diesem Zusammenhang eine Lösungsmöglichkeit bieten. Damit dies gelingt, könnte das Praxisteam dem Patienten bereits beim ersten Kontakt feste Termine in einer gedruckten Übersicht oder auf einer Terminkarte der Praxis für die beiden Folgequartale mitgeben. Zusätzlich sollte in der Praxis-EDV ein Filter für die Weiterbetreuung angelegt werden, der für das Praxisteam regelmäßig zu Beginn des Quartals Recall-Listen erstellt. Dann können Patienten an vereinbarte Termine erinnert werden. Oder aber eben daran, dass ein Kontrolltermin wieder sinnvoll wäre.

Aber Achtung: Für Terminerinnerungen benötigen Sie die Einverständniserklärung des Patienten. Diese lassen Sie sich am besten - schriftlich - gleich beim Ausfüllen des Anamnesebogens beim ersten Praxiskontakt geben. Hier sollten Sie zudem direkt abfragen, wie der Patient erinnert werden möchte: per EMail, Brief, Anruf oder SMS. Und an was die Praxis erinnern darf: Kontrolltermine, ausgemachte Termine oder auch anstehende Gesundheitsuntersuchungen. 